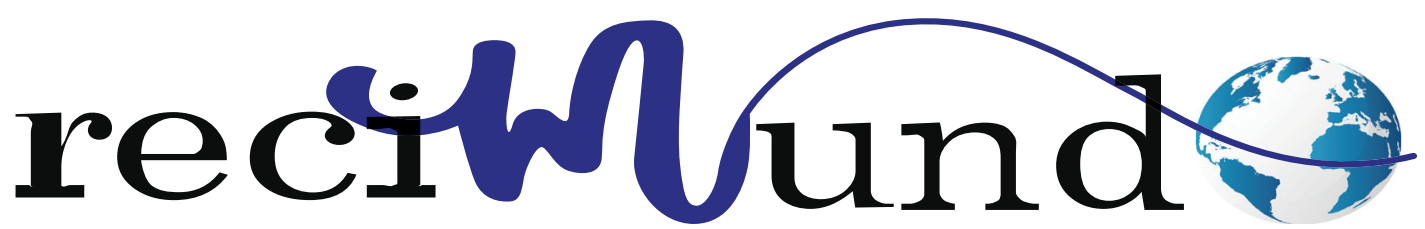

Revista Científica Mundo de la Investigación y el Conocimiento

DOI: 10.26820/recimundo/5.(2).julio.2021.249-256

URL: https://recimundo.com/index.php/es/article/view/1258

EDITORIAL: Saberes del Conocimiento

REVISTA: RECIMUNDO

ISSN: 2588-073X

TIPO DE INVESTIGACIÓN: Artículo de revisión

CÓDIGO UNESCO: 53 Ciencias Económicas

PAGINAS: 249-256

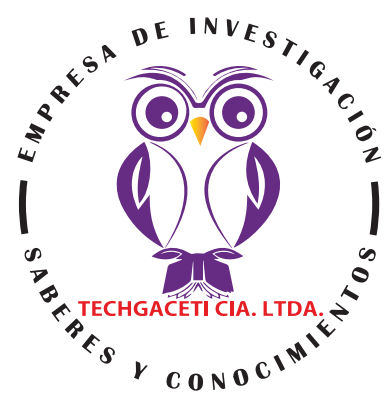

\title{
La visión de las PYMES manufactureras del Ecuador
}

The vision of manufacturing SMEs in Ecuador

A visão das PMEs manufatureiras no Equador

Magdalena Valero Camino'; Hugo Efraín Hidalgo Hidalgo2; Patricia Geovanna Guevara Córdova ${ }^{3}$

RECIBIDO: 11/04/2021 ACEPTADO: 15/06/2021 PUBLICADO: 30/07/2021

1. Master en Administración y Dirección de Empresa; Doctorando de la Universidad Andina Simón Bolívar Sede Sucre Bolivia; Docente de la Universidad Estatal de Bolívar; Ecuador; mvalero@ueb.edu.ec - valeromagdalena@gmail.com; (iD https://orcid.org/0000-0001-6001-4779

2. Máster Administración y dirección de empresas; Máster en Diseño Curricular; Universidad Estatal de Milagro; Unidad Educativa José María Velasco Ibarra; hhidalgoh@unemi.edu.ec - hugo.hidalgo@educacion.gob.ec; (iD https://orcid.org/00000001-8489-0685

3. Magister en Diseño Curricular; Diploma Superior en Diseño Curricular por Competencias; Profesor en Comercio y Administración; Licenciada en Ciencias de la Educación Especialización Comercio y Administración; Profesora de Segunda Enseñanza Especialización Comercio y Administración; Ingeniera Comercial Mención Administración Financiera; Ingeniera en Contaduría Pública y Auditoria CPA; Unidad Educativa Otto Arosemena Gómez; patricia.guevara@educacion.gob.ec;

(iD) https://orcid.org/0000-0001-8938-6971

\author{
CORRESPONDENCIA \\ Magdalena Valero Camino \\ mvalero@ueb.edu.ec \\ Bolívar; Ecuador
}




\section{RESUMEN}

La humanidad se encuentra experimentando una de las crisis sanitarias más grandes de todos los tiempos, la cual ha impactado profundamente todos los sectores, generada por la Pandemia del Covid-19. Esta situación ha llevado a los gobiernos a tomar decisiones que impactan diversos órdenes de la vida social y económica. Entre las medidas aplicadas, una de las que más impacto ha tenido es el confinamiento, el cual afecta de manera directa la economía, en todos los niveles, pero, especialmente se ven afectadas las pequeñas y medianas empresas (Pymes), ya que no cuentan con el capital y planes de financiamiento de las grandes empresas o corporaciones. Las Pymes desempeñan un papel muy importante en la economía de Ecuador, y han contribuido al desarrollo tanto económico como social, de acuerdo a las condiciones de mercado y a los cambios en la demanda, al tiempo que incentivan la industria nacional y disminuyen el índice de desempleo. El objetivo de la presente investigación consiste en plasmar la visión que se tiene de las Pymes manufactureras en Ecuador. El modelo de investigación es una revisión de tipo documental bibliográfico. Se encontró que la adaptación de las empresas a la nueva modalidad de comercio electrónico, ha constituido un verdadero nuevo reto, que demanda brindar todas las herramientas necesarias a los empleados y trabajadores para adaptarse a esta nueva modalidad de trabajo. Por último, es importante resaltar que el apoyo del gobierno del Ecuador es fundamental para las Pymes manufactureras. Alcanzar los resultados que se plantea este sector empresarial requiere una inyección de créditos con tasas accesibles de interés, que les permita mantener la resiliencia e innovación para su supervivencia, sobre todo luego del impacto de la pandemia del Covid-19.

Palabras clave: Pymes, manufactureras, visión, covid-19, empresas.

\section{ABSTRACT}

Humanity is experiencing one of the greatest health crises of all time, which has profoundly impacted all sectors, generated by the Covid-19 Pandemic. This situation has led governments to make decisions that impact various orders of social and economic life. Among the measures applied, one of those that has had the most impact is confinement, which directly affects the economy, at all levels, but especially small and medium-sized enterprises (SMEs) are affected, since they do not have with the capital and financing plans of large companies or corporations. SMEs play a very important role in Ecuador's economy, and have contributed to both economic and social development, according to market conditions and changes in demand, while encouraging national industry and reducing the unemployment rate. . The objective of this research is to capture the vision of manufacturing SMEs in Ecuador. The research model is a bibliographic documentary type review. It was found that the adaptation of companies to the new modality of electronic commerce has constituted a real new challenge, which demands to provide all the necessary tools to employees and workers to adapt to this new modality of work. Finally, it is important to highlight that the support of the Ecuadorian government is essential for manufacturing SMEs. Achieving the results proposed by this business sector requires an injection of credits with accessible interest rates, which allows them to maintain resilience and innovation for their survival, especially after the impact of the Covid-19 pandemic.

Keywords: Smes, manufacturers, vision, covid-19, companies.

\section{RESUMO}

A humanidade está passando por uma das maiores crises de saúde de todos os tempos, que afetou profundamente todos os setores, gerada pela Pandemia Covid-19. Essa situação tem levado governos a tomar decisões que impactam várias ordens da vida social e econômica. Dentre as medidas aplicadas, uma das que tem mais impacto é o confinamento, que afeta diretamente a economia, em todos os níveis, mas principalmente as pequenas e médias empresas (PMEs) são afetadas, uma vez que não têm com o capital e planos de financiamento de grandes empresas ou corporações. As PMEs desempenham um papel muito importante na economia do Equador, contribuindo para o desenvolvimento econômico e social, de acordo com as condições do mercado e as mudanças na demanda, ao mesmo tempo em que estimulam a indústria nacional e reduzem a taxa de desemprego. . O objetivo desta pesquisa é capturar a visão das PMEs manufatureiras no Equador. O modelo de pesquisa é uma revisão bibliográfica do tipo documental. Constatou-se que a adaptação das empresas à nova modalidade de comércio eletrônico tem se constituído em um verdadeiro novo desafio, que exige disponibilizar todas as ferramentas necessárias para que os funcionários e trabalhadores se adaptem a esta nova modalidade de trabalho. Finalmente, é importante destacar que o apoio do governo equatoriano é essencial para as PMEs manufatureiras. Alcançar os resultados propostos por este setor empresarial exige uma injeção de créditos com taxas de juros acessíveis, o que Ihes permite manter a resiliência e a inovação para sua sobrevivência, principalmente após o impacto da pandemia Covid-19.

Palavras-chave: Pmes, fabricantes, visão, covid-19, empresas. 


\section{Introducción}

Se conoce como Pymes al conjunto de pequeñas y medianas empresas que de acuerdo a su volumen de ventas, capital social, cantidad de trabajadores, y su nivel de producción o activos presentan características propias de este tipo de entidades económicas. Por lo general "en Ecuador las pequeñas y medianas empresas realizan diferentes tipos de actividades económicas entre las que destacamos las siguientes: comercio al por mayor y al por menor, agricultura, silvicultura y pesca, industrias manufactureras, construcción, transporte, almacenamiento, comunicaciones y otras" Andrade et al. (2016)

Las pequeñas y medianas empresas (Pymes) representan un parte importante de la economía de un país, y son determinantes en la generación de ingresos y fuentes de empleos de una nación. De acuerdo a lo referido por Solis \& Robalino (2019):

\begin{abstract}
"Alrededor del $80 \%$ de las empresas constituidas en el Ecuador corresponden a Pymes y ésta conducta se observa en los demás países del mundo, pero lamentablemente se encuentran deficiencias en la gestión administrativa lo que ocasiona que ante la sociedad sean consideradas como empresas que no maduran económicamente".
\end{abstract}

Las Pymes constituyen en el Ecuador la principal fuente del desarrollo social en cuanto a producción, demanda y compra de productos o simplemente por valor agregado, lo que significa que se ha convertido en un factor indispensable para generar riqueza y empleo.

En este sentido, Yance et al. (2017) hace referencia a investigación desarrollada por el Instituto Nacional de Estadísticas y Censos
(INEC), indicando que "estas empresas se caracterizan por el uso intensivo de la mano de obra, poca aplicación de la tecnología, baja división del trabajo, reducido capital, baja productividad, mínima capacidad de ahorro y limitado uso de los servicios financieros y no financieros."

La importancia de las pymes manufactureras en Ecuador radica en que son empresas que por su flexibilidad y bajos volúmenes de negocios, aportan grandes beneficios a la economía, permitiendo descentralizar totalmente la mano de obra, consiguiendo un mercado de trabajo seguro y cumpliendo así un buen papel dentro del mercado empresarial.

El objetivo de la presente investigación es plasmar la visión que se tiene de las Pymes manufactureras en Ecuador, destacando la necesidad de adaptación a la nueva modalidad de trabajo basada en el comercio electrónico y al impacto de las políticas que aplique el gobierno de Ecuador y que permitan la sostenibilidad y desarrollo de estas empresas en el mediano y largo plazo.

\section{Materiales y Métodos}

Para el desarrollo de la presente investigación se utilizaron ordenadores con conexión a internet, por medio de los cuales se ubicó el material bibliográfico base para realizar el análisis y compendio de ideas. En tal sentido, se clasifica esta investigación como de tipo documental o bibliográfica.

Se usaron buscadores como Google y Google Académico. Entre las páginas usadas podemos mencionar economipedia, scielo, eumed, el sitio web del Instituto Nacional de Estadísticas y Censos, entre otras.

Fueron incluidos materiales tales como informes, tesis de grado y otras clases de contenidos. Contenidos repetidos, las editoriales o cartas editoriales, anotaciones académicas y todo documento carente de

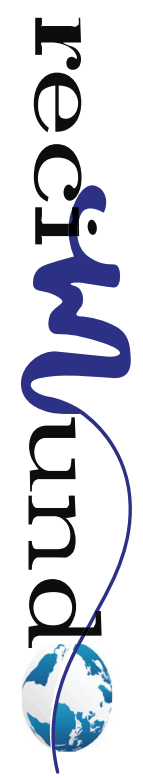


sustento científico o bajo nivel de evidencia fueron excluidos de la selección.

En cuanto al tiempo de investigación, esta se llevó a cabo durante los primeros días del mes de junio de 2021. Los descriptores usados fueron los siguientes: "Pymes manufactureras en ecuador", y "Visión de las Pymes manufactureras en Ecuador". Igualmente, la información encontrada se fue filtrando bajo algunos criterios tales como idioma, el cual se usó el español, mayor relevancia y con correlación del tema. Los trabajos seleccionados se encontraron dentro del rango de fecha de publicación, destacando los del 2020 al 2021.

Por último, se resalta que, tanto la metodología como el análisis y la argumentación que se expusieron en la presente investigación, fueron llevadas a cabo en equipo y de manera consensuada entre todos los investigadores.

\section{Resultados}

Pymes, es el acrónimo utilizado a la hora de hablar de Pequeñas y Medianas Empresas. Estas, generalmente suelen contar con un bajo número de trabajadores y un volumen de negocio e ingresos moderados en comparación con grandes corporaciones industriales o mercantiles.

Se puede plantear que no existe un concepto o definición exacta de Pymes, ya que las variables de estas pequeñas y medianas empresas pueden cambiar de acuerdo con la economía del país donde producen. "Una Pyme puede ser grande para un país cuya economía no alcanza niveles significativos en relación de la economía mundial, mientras que para otro país con una economía grande la propia empresa puede ser pequeña" (Rodríguez \& Aviles, 2020).

Por su parte Baque et al. (2020) indica lo siguiente:
Son organizaciones con fines de lucro (que buscan generar beneficios) y que cuyas operaciones son de baja escala. Aunque el concepto de Pyme queda bastante reducido a una cuantificación de producción y trabajadores, dependiendo del territorio en el que se encuentran se puede calificar a una empresa como tal entorno a una visión subjetiva de los índices a los que este sujeto, en otras palabras, depende de la zona geográfica en la que se establecen se toma como referencia para determinar el número de trabajadores máximos o el grado de sus operaciones que determinen a una empresa como Pymes o no.

\section{Origen de las PYMES en Ecuador}

Las Pymes surgen en la década de 1950, destacándose en la producción de tejidos, madera y alimentos, al tiempo que generaban fuentes de empleo y contribuían a reducir el índice de pobreza (Gudiño, 2017).

En sus comienzos este sector se vio afectado por condiciones limitantes para su desarrollo, como, escasos planes de apoyo y falta de normas legales que impedían la conformación de estas empresas.

En la actualidad las Pymes desempeñan un papel muy importante en la economía de Ecuador, y han contribuido al desarrollo tanto económico como social, de acuerdo a las condiciones de mercado y a los cambios en la demanda al tiempo que incentivan la industria nacional y disminuyen el índice de desempleo.

De acuerdo a censo realizado en el año 2017 por el Instituto Nacional de Estadísticas y Censos (INEC) de Ecuador, citado por Useche et al. (2021) "se erigen como dinamizadoras de la economía pues, en ese país el 99\% de los negocios se desempeñan bajo esta modalidad empresarial". 


\section{Clasificación de las PYMES en Ecuador}

Tradicionalmente las empresas se clasifican según su tamaño en pequeñas, medianas y grandes. Así pues, al conjunto de las dos primeras se le denomina de forma abreviada Pymes (pequeñas y medianas empresas). Además, con el paso de los años se les ha sumado a estos tres grupos un cuarto: las microempresas, que también se incluyen en las Pymes.

\section{Clasificación Trabajadores Ventas anuales (USD)}

\begin{tabular}{lll}
\hline Microempresa & $1-9$ & Iguales o menores a $\$ 100.000$ \\
\hline Pequeña empresa & $10-49$ & Entre 100.001 a $1^{\prime} 000.000$ \\
\hline Mediana empresa & $50-199$ & Entre $\$ 1^{\prime} 000.000$ y $5^{\prime} 000.000$ \\
\hline
\end{tabular}

Figura 1. Clasificación de las micro, pequeñas y medianas empresas en Ecuador.

Fuente: Las micro, pequeñas y medianas empresas. Chávez et al. (2018) Conrado 14(1). Recuperado de: http://scielo.sld.cu/scielo.php?script=sci_arttext\&pi$d=S 1990-86442018000500247$

Las Pymes por ser empresas con bajo volumen de trabajadores e ingresos en comparacion con las grandes corporaciones, se caracterizan por ser flexibles y adaptarse a los constantes cambios que vivimos actualmente, sin embargo, estas mismas caracteristicas hace que sean empresas con debilidades de carácter estructural en su gestión y una inadecuada asignación de sus recursos.

En este sentido Westreicher (2015) cita las que considera ventajas y desventajas mas relevantes de este tipo de empresas:

- Ventajas:

- Es más sencillo que puedan cambiar el nicho o modelo de negocio. Es decir, existe mayor flexibilidad.

- La relación entre el empresario y sus clientes es cercana. Esto, ya que usualmente el trato es directo entre ambos.

- Lo anterior genera un vínculo entre el negocio y los clientes que se traduce muchas veces en fidelidad.

- Son empresas que pueden encontrar nichos de mercado no atendidos.
- Desventajas:

- Al no tener un gran volumen de transacciones no alcanzan economías de escala. Es decir, sus operaciones podrían tener un menor coste unitario si el número de ventas fuera mayor.

- Es más complicado para una pyme conseguir financiamiento, en comparación a una gran empresa. Esto, en vista que sus ingresos y su respaldo financiero son menores.

- En caso que la pyme acceda a un crédito, probablemente sea a un tipo de interés alto, en comparación al que debe pagar en promedio una gran corporación.

- Puede resultar más difícil invertir en campañas publicitarias masivas o en desplegar una extensa red de ventas.

\section{Importancia de las PYMES en Ecuador}

Las Pymes constituyen un factor importante para el crecimiento socio-económico de cada país, lo que da origen a la necesidad de incrementar su desempeño y requerir la implementación de estrategias que benefi-

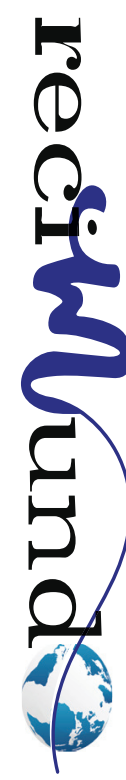


cien las operaciones, todo con la finalidad de reducir los costos de operación, mejorar la eficiencia de los procesos, los niveles de inventario, la calidad de los productos y por supuesto incrementar la productividad.

Las pymes en el Ecuador contribuyen a su desarrollo socioeconómico con aportaciones a la producción y distribución de bienes y servicios, a través de la transformación de la matriz productiva, ayudando a incrementar su desempeño generando empleo que dinamiza la economía y mejoran la gobernabilidad.

Según Sumba et al. (2020) la importancia de las Pymes ecuatorianas radica en que "son empresas que aportan a la economía, permitiendo descentralizar totalmente la mano de obra consiguiendo un mercado de trabajo seguro y cumpliendo así un buen papel dentro del mercado empresarial".

\section{Visión de las PYMES en Ecuador}

La visión de una empresa significa la imagen de a dónde se quiere llegar, así como de cómo desea verse la organización en un período de tiempo meta. De esta manera se plantea el futuro que se desea. En este sentido la empresa debe responder qué trata de conseguir, por medio de qué valores, de qué manera producir estos resultados que se esperan, de qué manera enfrentar los cambios que se presenten y cómo seguir siendo competitivos.

En el último año las empresas a nivel mundial han tenido que enfrentarse a una pandemia por el Covid-19, lo que ha desafiado al sistema socioeconómico mundial, y las Pymes manufactureras ecuatorianas, no escapan de esta situación, viéndose afectadas por la paralización de sus actividades productivas debido a la contracción de la demanda por confinamiento social, por lo que se han visto en la obligación de repensar sus modelos de negocio.
En tiempos de Covid-19 el uso de la tecnología se ha vuelto prácticamente un requisito obligatorio a nivel empresarial, más si se trata de actividades comerciales. Si una empresa no se adapta a las tendencias actuales, fácilmente terminaría perdiendo su mercado.

En este sentido Ortiz et al. (2020) refiere que:

A corto plazo, el comercio electrónico en Ecuador ha permitido a las Pymes mantener en funcionamiento sus cadenas de suministro evitando el contacto en persona. A mediano y largo plazo se estima que el comercio electrónico ayudará a efectuar transacciones más allá de las fronteras de los estados y países al ofrecer plataformas a las que se puede acceder en todas las geografías. Esta facilidad de acceso es fundamental a la luz de la escasez de liquidez y la disminución de la capacidad financiera de compradores y productores.

Por su parte, Betancourt et al. (2020) considera que las empresas deben hacer un replanteamiento de las técnicas de gestión empresarial, entre las que destaca las siguientes:

- Análisis estratégico: realiza un diagnóstico del contexto político, económico y social a nivel local, nacional e internacional.

- Gestión Organizacional o Proceso Administrativo: planifica anticipadamente el quehacer futuro de la organización, fijando estrategias, metas y objetivos a cumplir.

- Gestión de la Tecnología de la Información y Comunicación: aplica los sistemas de información y comunicación internos y externos a la empresa.

- Gestión Financiera: obtiene los recursos económicos y créditos con el menor costo posible para luego asignarlos, controlarlos y evaluarlos, buscando el máximo rendimiento de la empresa. 
- Gestión de Recursos Humanos: utiliza la fuerza laboral de la forma más eficiente.

El apoyo del gobierno del Ecuador es fundamental para las Pymes manufactureras. Alcanzar los resultados que se plantea este sector empresarial requiere una inyección de créditos con tasas accesibles de interés que les permita mantener la resiliencia e innovación para su supervivencia, sobre todo luego del impacto de la Pandemia del Covid-19. En tal sentido, es importante resaltar lo siguiente:

El gobierno del Ecuador ha puesto en marcha una serie de programas a lo largo de esta Pandemia que pretenden dar el apoyo necesario a las Pymes de la región. El programa Resurgimos Ecuador, que reemplaza a Reactívate Ecuador, a partir de abril de 2021, permite que las micro, pequeñas, medianas empresas, artesanos y actores de la economía popular y solidaria que facturan hasta $\$ 5$ millones puedan acceder a créditos de hasta $\$ 500.000$ con plazos de pago de 48 meses y seis meses de gracia. (El Universo, 2021)

Con la implantación de estas estrategias, se intenta conseguir una activación inmediata de las Pymes, rompiendo con la modalidad de trabajo tradicional y atreviéndose a insertarse en nuevos espacios que implicarán más que retos, grandes aprendizajes y otra visión del negocio y de la manera de gerenciar; pero, sin duda alguna, también conllevarán otro nivel de competencia y desenvolvimiento en el mercado nacional e internacional.

\section{Conclusión}

Algunos aspectos del entorno de las Pymes inciden en forma bastante determinante en el desarrollo, entre ellos cabe destacar: la estabilidad y las políticas macroeconómicas más en sintonía con el desarrollo económico; los programas de fomento y otros factores existentes que promueven su desarrollo.

El marco regulatorio que les afecta y el grado de formalización de las empresas son factores que inciden en la competitividad como los relacionados con el sistema educativo, la capacitación, la investigación y el desarrollo tecnológico.

Para finalizar consideramos que es necesaria una mayor cohesión social de todos los actores que mediante el diálogo puedan mejorar las dificultades actuales que presentan estas empresas.

\section{Bibliografía}

Andrade, C., Iriarte, M., \& Zambrano, J. (22 de 09 de 2016). Caracterización de las MIPYMES cantón Flavio Alfaro, Provincia Manabí, Ecuador. Dominio de las ciencias, 2(4), 461-471. Recuperado el 02 de 06 de 2021, de file:///C:/Users/Brandon/Downloads/269-1003-1-PB.pdf

Baque, M., Cedeño, B., Chele, J., \& Gaona, V. (12 de 2020). Fracaso de las pymes: Factores desencadenantes, Ecuador 2020. FIPCAEC, 5(4), 3-25. doi:https://doi.org/10.23857/fipcaec.v5i4.293

Betancourt, F., Ollague, J., Pacheco, A., \& Tapia , N. (11 de 12 de 2020). La gestión empresarial ante la crisis del covid-19 y el escenario futuro en las Py-MEs del cantón Arenillas, provincia de El Oro, Ecuador. 593 Digital Publisher, 5(6), 496-514. doi:doi.org/10.33386/593dp.2020.6-1.447

Chávez, G., Campuzano, J., \& Betancourt, V. (03 de 12 de 2018). Las micro, pequeñas y medianas empresas. Clasificación para su estudio en la carrera de Ingeniería en Contabilidad y Auditoría de la Universidad Técnica de Machala. Conrado, 14(1). Recuperado el 03 de 06 de 2021, de http:// scielo.sld.cu/scielo.php?script=sci_arttext\&pid=S1990-86442018000500247 
El Universo. (20 de Abril de 2021). Gobierno lanza programa Resurgimos Ecuador, que ofrece créditos de hasta $\$ 500.000$ para mipymes. El Universo. Recuperado el 21 de Julio de 2021, de https:// www.eluniverso.com/noticias/economia/gobierno-lanza-programa-resurgimos-ecuador-que-ofrece-creditos-de-hasta-500000-para-mipymes-nota/

Gudiño, R. (21 de 11 de 2017). Universidad Veracruzana. Recuperado el 04 de 06 de 2021, de https:// www.uv.mx/iiesca/files/2018/03/14CA201702.pdf

Ortiz, O., Rodriguez., K., Quiroz, A., \& Parrales, M. (05 de 11 de 2020). El e-commerce y las Mipymes en tiempos de Covid-19. Espacios, 41(42), 100-117. doi:ISSN: 0798-1015 DOI: 10.48082/espacios-a20v41n42p09

Rodríguez, R., \& Aviles, V. (09 de 2020). Las PYMES en Ecuador. Un analisis necesario. 593 Digital Publisher, 5(5), 191-200. doi:https://doi.org/10.33386/ 593dp.2020.5-1.337

Solis, L., \& Robalino, R. (12 de 2019). El papel de las PYMES en las sociedades y su problemática empresarial. INNOVA Research Journal, 4(3), 85-93. doi:https://doi.org/10.33890/innova. v4.n3.2019.949
Sumba, Y., Cardenas, N., Bravo, T., \& Arteaga, R. (12 de 2020). La planeación estratégica: Importancia en las PYMES ecuatorianas. Ciencias Economicas y Empresariales, 5(4), 114-136. doi:https://doi. org/10.23857/fipcaec.v5i4.299

Useche, M., Vasquez, L., Vázquez, F., \& Ordóñez, M. (2021). Fórmula estratégica empresarial para pymes en Ecuador ante la covid-19. Universidad \& Empresa, 23(40), 1-22. doi:https://doi. org/10.12804/revistas. urosario.edu.co/empresa/a.9309

Westreicher, G. (27 de 06 de 2015). Economipedia. Recuperado el 02 de 06 de 2021, de Economipedia: https://economipedia.com/definiciones/pyme. $\mathrm{html}$

Yance, C., Solis , L., Burgos , I., \& Hermida, L. (06 de 2017). Enciclopedia Virtual EUMED. Recuperado el 05 de 06 de 2021, de Enciclopedia Virtual EUMED: https://www.eumed.net/cursecon/ecolat/ ec/2017/pymes-ecuador.html

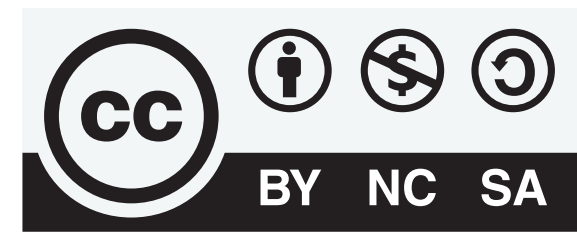

CREATIVE COMMONS RECONOCIMIENTO-NOCOMERCIAL-COMPARTIRIGUAL 4.0.

\section{CITAR ESTE ARTICULO:}

Valero Camino, M., Hidalgo Hidalgo, H. E., \& Guevara Córdova, P. G. (2021). La visión de las PYMES manufactureras del Ecuador. RECIMUNDO, 5(3), 249256. https://doi.org/10.26820/recimundo/5.(2).julio.2021.249-256 